\title{
Die Schweizerische Transplantationskohortenstudie (STCS)
}

\author{
Michael T. Kollera, \\ Christian van Delden ${ }^{b}$, \\ Manuel Pascualc, \\ Nicolas Müller', \\ Philippe Baumanne, \\ Jürg Steiger $f_{\text {im Namen aller }}$ \\ Mitglieder der Schweizerischen \\ Transplantationskohorten- \\ studie*
}

a Basel Institut für klinische Epidemiologie und Biostatistik, Universitätsspital Basel (BICE)

b Service de Transplantation, HUG

c Centre de transplantation, CHUV

d Klinik für Infektionskrankheiten und Spitalhygiene, Universitätsspital Zürich

e Service d'informatique médicale, Unité d'informatique clinique, Hôpitaux Universitaires de Genève

f Transplantationsimmunologie und Nephrologie, Universitätsspital Basel

* siehe Kasten auf S. 955.

Korrespondenz:

Prof. Dr. med. Jürg Steiger

Transplantationsimmunologie und Nephrologie

Universitätsspital Basel

Petersgraben 4

CH-4031 Basel

jsteiger@uhbs.ch

\section{Transplantation in der Schweiz}

Die Transplantationsmedizin hat in der Schweiz seit der ersten Nierentransplantation im Jahre 1964 eine langjährige Tradition. In Basel wurde 1973 die allogene Stammzelltransplantation und 1986 die autologe Stammzelltransplantation eingeführt und entwickelt in Bern begann man 1978 mit autologen Stammzelltransplantationen. Nach der ersten Lebendnierentransplantation im Jahre 1967 begann in Basel die Spezialisierung in den Nierentransplantationen. In Bern und Zürich wurden 1983 und 1986 die ersten Lebertransplantationen durchgeführt. Zürich war wegweisend in der Entwicklung der Herztransplantation seit den 80er Jahren sowie der Lungentransplantation seit Beginn der 90er Jahre. Genf hat sich seit Ende der 80er Jahre auf Transplantationen bei Kindern spezialisiert und hat die Spezialität Dünndarmtransplantation und erste Multiorgantransplantation durchgeführt. Die Transplantationszentren Lausanne und Genf arbeiten seit 2004 im Rahmen eines «Centre romand de transplantation» eng zusammen. So etwa werden Lungen und Herzen nur noch in Lausanne transplantiert und abdominelle Organe wie Leber, Bauchspeicheldrüse oder Dünndarm in Genf. Am Kantonsspital St. Gallen wurde im Januar 1969 die ersten Nierentransplantationen durchgeführt. Die Durchführung an einem nichtuniversitären Zentrum galt damals als grosser Fortschritt. Im Jahre 1992 wurde zudem in St. Gallen erstmals eine Lebendnierentransplantation durchgeführt. Die allogene Stammzelltransplantation wird heute in Basel, Genf und Zürich, die autologe in Aarau, Basel, Bellinzona, Bern, Lausanne, St. Gallen und Zürich durchgeführt.

Die Transplantationsmedizin hat in der Schweiz in den letzen 25 Jahren einen hohen technischen Standard erreicht. Zusammen mit SwissTransplant, der nationalen Koordinationszentrale der Organallokation, werden Organe aus dem In- und Ausland alloziert. Stammzelltransplantate von nichtverwandten Spendern werden über die Landesgrenzen alloziert und von der Stiftung Swiss Blood Stem Cells koordiniert. Nabelschnurbluttransplantate werden von der Schweizerischen Nabelschnurblutbank Swisscord in Basel und Genf koordiniert.

\section{Transplantationszahlen in der Schweiz}

In der Schweiz werden jährlich etwa 400-500 solide Organe und etwa 300-400 Stammzelltransplantationen durchgeführt. Bei den soliden Organen kommt die Nierentransplantation (etwa 200-300/Jahr) vor der Lebertransplantation (80-100/Jahr) und den deutlich selteneren Herz- und Lungentransplantationen (etwa
25-40/Jahr). Pankreas, Inselzell- und Dünndarmtransplantationen kommen am seltensten vor. Hingegen ist die Nieren-und Pankreastransplantation die am häufigsten durchgeführte kombinierte Transplantation.

\section{Bestrebungen zu nationaler Harmonisation in der Transplantation}

Dank dem Einsatz multidisziplinärer Teams und grosser Forschungsbemühungen werden heutzutage z. B. bei der Nierentransplantation mittlere Organüberlebensraten von 10 bis 15 Jahren erreicht. Trotz dieser Fortschritte sind die verantwortlichen Ärzte täglich mit der Schwierigkeit konfrontiert, Transplantatempfängern ein optimales Management zu bieten. Dazu kommt, dass das neue Schweizerische Transplantationsgesetz alle transplantierenden Zentren dazu verpflichtet, Daten zur Behandlungsqualität transplantierter Patienten dem Bundesamt für Gesundheit (BAG) zu melden.

Die Daten der Patienten mit Stammzelltransplantationen werden in der Schweiz seit längerer Zeit durch die «Swiss Transplant Arbeitsgruppe Blood and Marrow Transplantation» (STABMT) gesammelt und die Transplantationszentren arbeiten nach einem einheitlichen europäischen Qualitätsstandard. Die Daten der soliden Organtransplantationen wurden bisher in lokalen Systemen gesammelt. Aus diesem Grund schlossen sich alle sechs Schweizer Transplantationskliniken (Basel, Bern, Genf, Lausanne, St.Gallen und Zürich) zusammen, um klinische Daten mit Laborproben zentral in einem Datenbanksystem zu erfassen. Im Rahmen des ersten Calls der Fachkommission Patientenorientierte Klinische Forschung (PaKliF) des Schweizerischen Nationalfonds (SNF) im Herbst 2004 wurde die Swiss Transplant Cohort Study (STCS) als Projekt eingereicht. Im November 2005 beschloss der SNF, das Projekt für mindestens drei Jahre zu fördern. Ebenfalls beschlossen alle Universitätsspitäler und das Kantonsspital St. Gallen, eine gemeinsame Qualitätskontrolle durchzuführen. Um dieses neue und wegweisende Vorgehen zu ermöglichen, finanzieren die obgenannten Spitäler ebenfalls die STCS mit. Somit wird die STCS durch die Spitäler im Sinne der Qualitätskontrolle unterstützt und die Unterstützung des Schweizerischen Nationalfonds ermöglicht wissenschaftliche Analysen aller in der Schweiz durchgeführten Transplantationen. Unter dieser Voraussetzung nahmen die organspezifischen Arbeitsgruppen zusammen mit Epidemiologen und IT-Spezialisten die grosse Arbeit zur Definition der STCS-Datensätze auf, welche auf den Vorarbeiten des «Centre romand de transplantation» basieren. 


\section{Die Schweizerische} Transplantationskohortenstudie (STCS)

Die STCS ist eine prospektive «offene» Kohortenstudie, die organübergreifend alle Transplantatempfänger, die ihre Zustimmung zur Mitwirkung geben, zum Zeitpunkt der Transplantation einschliesst. Patienten auf der Warteliste für solide Organe werden vor erfolgter Transplantation um ihre Einwilligung zur Partizipation angefragt, Patienten für eine Stammzelltransplantation zum Zeitpunkt der Transplantation. Die Ethikkommissionen aller partizipierenden Zentren haben Anfang 2008 die Studie gutgeheissen. Die STCS begann ab 2. Mai 2008 Patientinnen und Patienten einzuschliessen und prospektiv zu beobachten. Es ist wichtig zu bemerken, dass ein sogenannter minimaler Datensatz, der vom Gesetzgeber vorgeschrieben ist, bei allen Patienten erfasst werden muss. Mit der Zustimmung ermöglicht der Patient eine breitere Datenerfassung und auch das Asservieren von Blutproben.

Hauptziel der Studie ist, schweizweit eine vollständige Übersicht der Transplantationsaktivitäten, Resultate sowie angewandter Methoden zu gewinnen. Dies ist eine primäre Voraussetzung, um die Lebensqualität der Patienten sowie die Lebenserwartung von Transplantatempfängern und vom transplantierten Organ zu verbessern. Im Speziellen sind die Studienverantwortlichen an der Erforschung folgender Themen interessiert:

- Transplantationsinfektiologie;

- Transplantationsimmunologie;

- psychosoziale und verhaltenspsychologische Einflussfaktoren sowie Untersuchung der Lebensqualität in transplantierten Patienten;

- Langzeiteinflüsse der immunsuppressiven Therapie;

- Einfluss von genetischen Faktoren und Biomarkern auf Abstossung und Transplantatüberleben.

Jedes Zentrum hat speziell geschulte Datenmanager eingestellt, die zusammen mit den behandelnden Ärzten und Transplantationskoordinatoren die nötigen Daten erheben und den Follow-up der Patienten sicherstellen. Weiter sind mit dem Projekt assoziiert das Basel Institut für klinische Epidemiologie und Biostatistik der Universität Basel (BICE), das die STCS von epidemiologischer Seite unterstützt, sowie die Unité d'informatique clinique der Universität Genf (SIM), die die IT-Plattform entwickelt hat und unterhält. Die Daten der Stammzelltransplantation werden wie bisher durch das elektronische Datenerfassungssystem PROMISE der European Group for Blood and Marrow Transplantation (EBMT) erfasst.

Die STCS wird durch das Board of Representatives (BOR) geführt. Darin vertreten sind alle Transplantationszentren. Weiter nehmen Vertreter von SwissTransplant, der Pädiatrie und Swiss Blood Stem Cells Einsitz. Für die tägliche Arbeit und die Vorbereitung der Sitzungen ist ein Executive Office (EO) verantwortlich.
Das Scientific Committee behandelt die wissenschaftlichen Anträge, die an die STCS gestellt werden, und entscheidet, welche Projekte durchgeführt werden.

\section{STCS - ein komplexes Kohortenprojekt}

In der Transplantationsmedizin muss eine moderne prospektive Kohorte die Komplexität der Behandlung von Transplantatempfängern abbilden. Das heisst, es kommen nicht nur einzelne Organtransplantationen und deren prospektive Follow-ups vor, sondern auch komplizierte Szenarien wie simultane Transplantationen oder Retransplantationen.

Während der Phase des Kohortenaufbaus hat die «STCS Psychosocial Interest Group» einen transplantationsspezifischen psychosozialen Fragebogen, den sogenannten STCS Psychosocial Questionnaire (kurz PSQ) entwickelt. Es ist weltweit ein Novum, dass der Einfluss psychosozialer, sozioökonomischer und verhaltenspsychologischer Faktoren in Populationen unterschiedlicher Transplantatempfänger untersucht wird. Die Datenerhebung erfolgt bereits in der Phase vor der Transplantation und wird während regelmässiger Kohortenvisiten nach der Transplantation weitergeführt. Die Fragen stammen wo immer möglich aus validierten Instrumenten wie dem EuroQual (EQ5D), der Hospital Anxiety and Depression Scale (HADS) und dem Fragebogen zur Medikamententreue, der in der Schweizerischen HIV-Kohortenstudie validiert wurde.

Eine weitere Herausforderung stellte die Entwicklung eines Instruments zur Erfassung von Infektionskrankheiten dar, mit dem Pathogene und Infektlokalisationen detailliert über die Zeit registriert werden. Zusätzlich musste die Entwicklung eines Systems zur Registrierung und Monitorisierung von Blut- und DNA-Proben entwickelt werden, die zum Zeitpunkt der Transplantation sowie während den Follow-up-Untersuchungen den Patienten entnommen werden.

\section{Ausblick und Zusammenfassung}

In einer nächsten Phase werden allogene Stammzelltransplantationen der STCS angeschlossen. Eckdaten der STCS werden in regelmässigen Zeitabschnitten auf der Website der STCS (https://www.stcs.ch) sowie in wissenschaftlichen Zeitschriften veröffentlicht. Allerdings dauert es noch etwa 4 bis 5 Jahre bis erste Zahlen zum Langzeitüberleben von Spendern und transplantierten soliden Organen schweizweit verfügbar sein werden.

Die Studienverantwortlichen der STCS erhoffen sich, nebst wissenschaftlichem Fortschritt die Behandlung von transplantierten Patienten in der Schweiz nachhaltig zu verbessern und das Wirken in der Transplantationsmedizin transparenter zu gestalten. Durch eine zentrale Datenerfassung soll die Betreuung aller transplantierten Patienten in der Schweiz kontinuierlich kontrolliert und optimiert sowie ein Beitrag zu einem optimalen Nutzen der Ressourcen in der Transplantationsmedizin geleistet werden. 


\section{Mitglieder der Schweizerischen Transplantationskohortenstudie}

\section{Executive Office}

- Christian van Delden, Service de transplantation, Hôpitaux Universitaires de Genève

- Manuel Pascual, Centre de transplantation, CHUV Lausanne

- Jürg Steiger, Transplantationsimmunologie und Nephrologie, Universitätsspital Basel

Epidemiologie und Informatik

- Michael T. Koller und Heiner C. Bucher, Basel Institut für Klinische Epidemiologie und Biostatistik, Universitätsspital Basel (BICE)

- Philippe Baumann und Christian Lovis, Service d'informatique médicale, Unité d'informatique clinique, Hôpitaux Universitaires de Genève

\section{Zentrumsvertreter}

- Markus Weber, Klinik für Viszeral- und Transplantationschirurgie, Universitätsspital Zürich

- Nicolas Müller, Klinik für Infektionskrankheiten und Spitalhygiene, Universitätsspital Zürich

- Isabelle Binet, Nephrologie, Kantonsspital St. Gallen

- Pascal Meylan, Institut für Mikrobiologie, CHUV Lausanne

- Leo Bühler, Service de transplantation, Hôpitaux Universitaires de Genève

- Paul Mohacsi, Herz- und Gefässzentrum Bern, Inselspital Bern

- Hans-Peter Marti, Nephrologie und Hypertonie, Inselspital Bern

- Michael Dickenmann, Transplantationsimmunologie und Nephrologie, Universitätsspital Basel

\section{Stammzelltransplantation}

- Alois Gratwohl, Klinik für Hämatologie, Universitätsspital Basel

\section{Arbeitsgruppen STCS}

- Pädiatrische Transplantation: David Nadal, Infektiologie, Universitätskinderklinik Zürich (Leitung)

- Laborgruppe: Pascal Meylan, Institut für Mikrobiologie, CHUV Lausanne (Leitung)

- Datenmanagergruppe: Emmanuelle Catana, Centre de transplantation, CHUV Lausanne (Leitung)

- Psychosoziale Interessensgruppe: Sabina De Geest, Institut für Pflegewissenschaft, Universität Basel (Leitung)

- Infektiologie: Christian van Delden, Service de transplantation, Hôpitaux Universitaires de Genève (Leitung)

Arbeitsgruppen Swisstransplant

- Lunge: Annette Böhler, Pneumologie Universitätsspital Zürich

- Herz: Paul Mohacsi, Kardiologie Inselspital Bern

- Niere: Isabelle Binet, Nephrologie, Kantonsspital St. Gallen

- Leber: Christian Seiler, Viszeralchirurgie, Inselspital Bern

- Pankreas: Markus Müller, Klinik für Viszeral- und Transplantationschirurgie, Universitätsspital Zürich

- Inselzell- und Dünndarm: Thierry Berney, Service de Transplantation, Hôpitaux Universitaires de Genève

- Infektiologie: Christian van Delden, Service de transplantation, Hôpitaux Universitaires de Genève (Leitung)

Scientific Committee

- Nicolas Müller, Klinik für Infektionskrankheiten und Spitalhygiene, Universitätsspital Zürich (Leitung) 